\title{
Soluble Models of Strongly Interacting Ultracold Gas Mixtures in Tight Waveguides
}

\author{
M. D. Girardeau, ${ }^{1, *}$ and A. Minguzzi ${ }^{2}$, \\ ${ }^{1}$ College of Optical Sciences, University of Arizona, Tucson, AZ 85721, USA \\ ${ }^{2}$ Laboratoire de Physique et Modélisation des Mileux Condensés, C.N.R.S., B.P. 166, 38042 Grenoble, France
}

(Dated: October 25, 2018)

\begin{abstract}
A Fermi-Bose mapping method is used to determine the exact ground states of several models of mixtures of strongly interacting ultracold gases in tight waveguides, which are generalizations of the Tonks-Girardeau (TG) gas (1D Bose gas with point hard cores) and fermionic Tonks-Girardeau (FTG) gas (1D spin-aligned Fermi gas with infinitely strong zero-range attractions). We detail the case of a Bose-Fermi mixture with TG boson-boson (BB) and boson-fermion (BF) interactions. Exact results are given for density profiles in a harmonic trap, single-particle density matrices, momentum distibutions, and density-density correlations. Since the ground state is highly degenerate, we analyze the splitting of the ground manifold for large but finite BB and BF repulsions.
\end{abstract}

PACS numbers: 03.75.-b,03.75.Mn

Due to the rapidly increasing sophistication of experimental techniques for probing ultracold gases, theoretical emphasis has shifted from effective field approaches to more refined methods capable of dealing with correlations, and experiments measuring such correlations have been carried out. In ultracold gases confined in de Broglie waveguides with transverse trapping so tight that the atomic dynamics is essentially one-dimensional [1], with confinement-induced resonances [1, 2] allowing Feshbach resonance tuning [3] of the effective 1D interactions to very large values, such correlations are greatly enhanced. This has allowed experimental verification [4, 5] of the fermionization of bosonic ultracold vapors in such geometries predicted by the Fermi-Bose (FB) mapping method, which was introduced in 1960 [6] and used to obtain the exact $N$-particle ground and excited states of a $1 \mathrm{D}$ gas of impenetrable point bosons, the Tonks-Girardeau (TG) gas. Apart from recent work of Imambekov and Demler 7], theoretical work on 1D mixtures has used approximations which are not valid in the highly-correlated regime. Exact results in 7], based on the Bethe ansatz method of Lieb and Liniger (LL) 8], are limited to the spatially uniform case (no longitudinal trap potential). We present here exact solutions for several models of mixtures of strongly correlated 1D systems including that of [7], for both untrapped and harmonically trapped mixtures.

Bose-Fermi mixture: We consider a 1D mixture of $N_{B}$ bosons with point hard-core boson-boson interactions and of $N_{F}$ noninteracting fermions with point hardcore boson-fermion interactions, and we assume equal masses $m_{B}=m_{F}=m$. This model could be realized by choosing a bosonic and fermionic isotope of a given alkali element (e.g. $\left.{ }^{6} \mathrm{Li}-{ }^{7} \mathrm{Li}\right)$. We indicate the boson and fermion coordinates by $X_{B}=\left(x_{1 B}, \cdots, x_{N_{B} B}\right), X_{F}=$ $\left(x_{1 F}, \cdots, x_{N_{F} F}\right)$. Our treatment includes the case where the mixture is subjected to an external potential (e.g. a harmonic trap $v(x)=\frac{1}{2} m \omega^{2} x^{2}$ ), provided that the potentials acting on the bosons and on the fermions are the same, i.e., $v_{B}\left(x_{j B}\right)=v\left(x_{j B}\right)$ and $v_{F}\left(x_{\ell F}\right)=v\left(x_{\ell F}\right)$. The
Schrödinger Hamiltonian is $\hat{H}=\hat{H}_{B}+\hat{H}_{F}+\hat{H}_{B B}+\hat{H}_{B F}$ with

$$
\begin{aligned}
& \hat{H}_{B}=\sum_{j=1}^{N_{B}}\left[-\left(\hbar^{2} / 2 m\right) \partial^{2} / \partial x_{j B}^{2}+v\left(x_{j B}\right)\right] \\
& \hat{H}_{F}=\sum_{\ell=1}^{N_{F}}\left[-\left(\hbar^{2} / 2 m\right) \partial^{2} / \partial x_{\ell F}^{2}+v\left(x_{\ell F}\right)\right] .
\end{aligned}
$$

The impenetrable point $\mathrm{BB}$ and $\mathrm{BF}$ interactions can be formally represented as sums of LL delta function interactions [8] $g_{B B} \delta\left(x_{j B}-x_{\ell B}\right)$ and $g_{B F} \delta\left(x_{j B}-x_{\ell F}\right)$ with coupling constants $g_{B B} \rightarrow+\infty$ and $g_{B F} \rightarrow+\infty$, but they are more conveniently represented by constraints that the many-body wavefunction $\Psi\left(X_{B}, X_{F}\right)$ vanishes at all $\mathrm{BB}$ and $\mathrm{BF}$ collision points, after which $\hat{H}_{B B}$ and $\hat{H}_{B F}$ can be omitted from the Hamiltonian.

The exact solution can be obtained as follows. Construct a "model wavefunction" $\Psi_{M}\left(X_{B}, X_{F}\right)$ which is a Slater determinant of $N=N_{B}+N_{F}$ orthonormal orbitals $u_{1}(x), \cdots, u_{N}(x)$ occupied by all $N$ bosons and fermions, with all possible permutations of the atoms among the orbitals: $\Psi_{M}=\sum_{P} \varepsilon(P) u_{1}\left(P x_{1}\right) \cdots u_{N}\left(P x_{N}\right)$. Here $x_{1}, \cdots, x_{N}$ are $x_{1 B}, \cdots, x_{N_{B} B}, x_{1 F}, \cdots, x_{N_{F} F}$, the sum runs over all $N$ ! possible permutations of these variables including permutations exchanging bosons with fermions, and $\varepsilon(P)$ is the usual \pm 1 sign of the permutation. This wave function vanishes not only at the points $x_{j F}=x_{\ell F}$ required by fermionic antisymmetry, but also at the points $x_{j B}=x_{\ell B}$ and $x_{j B}=x_{\ell F}$ required by the $\mathrm{BB}$ and $\mathrm{BF}$ hard core constraints. Its improper symmetry under $\mathrm{BB}$ and $\mathrm{BF}$ exchange is repaired by a modified $\mathrm{FB}$ mapping. Define a mapping function $A\left(X_{B}, X_{F}\right)$ by

$$
A=\prod_{1 \leq j<\ell \leq N_{B}} \operatorname{sgn}\left(x_{j B}-x_{\ell B}\right) \prod_{j=1}^{N_{B}} \prod_{\ell=1}^{N_{F}} \operatorname{sgn}\left(x_{j B}-x_{\ell F}\right)
$$

where the $\operatorname{sign}$ function $\operatorname{sgn}(x)$ is $+1(-1)$ if $x>$ $0 \quad(x<0)$. Then the physical wavefunction is 
$\Psi\left(X_{B}, X_{F}\right)=A\left(X_{B}, X_{F}\right) \Psi_{M}\left(X_{B}, X_{F}\right) . \Psi_{M}$ is an exact many-body energy eigenstate if the orbitals $u_{\nu}$ are eigenfuctions of the single-particle Schrödinger Hamiltonian $-\left(\hbar^{2} / 2 m\right) \partial^{2} / \partial x^{2}+v(x)$ with eigenvalues $\epsilon_{\nu}$. Since $A$ is constant except for jumps at nodes of $\Psi_{M}$, it follows that the physical state $\Psi$ is an energy eigenstate with eigenvalue $\sum_{\nu} \epsilon_{\nu}$ and is symmetric under permutations of bosons and antisymmetric under permutation of fermions. The ground state is a filled Fermi sea of the lowest $N$ orbitals and excited states are generated by choosing higher orbitals. An important case for experiments is that of harmonic trapping, $v(x)=\frac{1}{2} m \omega^{2} x^{2}$. Then the ground state is a straightforward generalization of that of the trapped TG gas [9]. The orbitals are Hermite-Gaussians $\phi_{n}(x)=$ $\pi^{-1 / 4}\left(2^{n} n ! x_{\mathrm{osc}}\right)^{-1 / 2} e^{-Q^{2} / 2} H_{n}(Q)$ with $n=0,1, \cdots, N$, where $Q=x / x_{\mathrm{osc}}$ and $x_{\mathrm{osc}}=\sqrt{\hbar / m \omega}$. $\Psi_{0 M}$ can be reduced to Bijl-Jastrow form by determinantal algebra as in the original solution [ 6 ] for the untrapped TG gas and the recent one [9] for the trapped TG gas, yielding $\Psi_{0 M} \propto\left[\prod_{1 \leq j<\ell<N}\left(x_{j}-x_{\ell}\right)\right] \prod_{j=1}^{N} \phi_{0}\left(x_{j}\right)$ where $x_{1}=$ $x_{1 B}, \cdots, x_{N_{B}}=x_{N_{B} B}, x_{N_{B}+1}=x_{1 F}, \cdots, x_{N}=x_{N_{F} F}$. By applying the mapping function $A$ to $\Psi_{0 M}$ one finds for the ground state

$$
\begin{aligned}
& \Psi_{0}=\left[\prod_{1 \leq j<\ell \leq N_{B}}\left|x_{j B}-x_{\ell B}\right|\right]\left[\prod_{j=1}^{N_{B}} \prod_{\ell=1}^{N_{F}}\left|x_{j B}-x_{\ell F}\right|\right] \\
& \times\left[\prod_{1 \leq j<\ell \leq N_{F}}\left(x_{j F}-x_{\ell F}\right)\right] \phi_{0}\left(x_{1 B}\right) \cdots \phi_{0}\left(x_{N_{B} B}\right) \\
& \times \phi_{0}\left(x_{1 F}\right) \cdots \phi_{0}\left(x_{N_{F} F}\right) .
\end{aligned}
$$

apart from normalization. The solution for the homogeneous system of size $L$ with periodic boundary conditions differs only by omission of the Gaussian factors $\phi_{0}$ and by replacement of each coordinate difference $x-x^{\prime}$ by $\sin \left[\pi\left(x-x^{\prime}\right) / L\right]$ [6]. The bosonic and fermionic reduced one-body density matrices are defined as $\rho_{1 B}\left(x, x^{\prime}\right)=$ $\mathfrak{N}_{B} \int \Psi_{0}\left(x, X_{B}^{\prime}, X_{F}\right) \Psi_{0}^{*}\left(x^{\prime}, X_{B}^{\prime}, X_{F}\right) d X_{B}^{\prime} d X_{F} \quad$ and $\rho_{1 F}\left(x, x^{\prime}\right)=\mathfrak{N}_{F} \int \Psi_{0}\left(X_{B}, X_{F}^{\prime}, x\right) \Psi_{0}^{*}\left(X_{B}, X_{F}^{\prime}, x^{\prime}\right) d X_{B} d X_{F}^{\prime}$, where $X_{B}^{\prime}=\left(x_{2 B}, \cdots, x_{N_{B} B}\right)$ and $X_{F}^{\prime}=$ $\left(x_{1 F}, \cdots, x_{N_{F}-1, F}\right)$, and $\mathfrak{N}_{B}, \mathfrak{N}_{F}$ are normalization constants fixed by the conditions $\int \rho_{1 B}(x, x) d x=N_{B}$ and $\int \rho_{1 F}(x, x) d x=N_{F}$.

Density profiles: The bosonic and fermionic singleparticle densities $\rho_{B}(x) \equiv \rho_{1 B}(x, x)$ and $\rho_{F}(x) \equiv$ $\rho_{1 F}(x, x)$ are the same as those of $\Psi_{0 M}$, since they depend only on $\left|\Psi_{0}\right|^{2}$, and $A^{2}=1$. Since $\Psi_{0 M}$ is completely antisymmetric under permutations of all $N$ particles, $\rho_{B}$ and $\rho_{F}$ are both proportional to the density $\rho_{\mathrm{TG}}$ of a harmonically trapped TG gas of $N$ bosons: $\rho_{B}(x)=\left(N_{B} / N\right) \rho_{\mathrm{TG}}(x)$ and $\rho_{F}(x)=\left(N_{F} / N\right) \rho_{\mathrm{TG}}(x)$ where [9] $\rho_{\mathrm{TG}}(x)=\sum_{n=0}^{N-1}\left|\phi_{n}(x)\right|^{2}$. These exact results contrast strongly with local density approximation results in the TG limit [7], which show BF phase separation with $\rho_{B}$ more concentrated in the center, although their total density $\rho_{B}(x)+\rho_{F}(x)$ agrees closely with ours. Note that the exact ground state is highly degenerate in the TG limit [10] due to the fact that there is no required symmetry under BF exchange. Our choice (3) is the most symmetrical, has hard-core cusps at all BB and $\mathrm{BF}$ collisions and antisymmetry nodes at all FF collisions, and it will be shown below that if the degeneracy is lifted by making the $\mathrm{BB}$ and $\mathrm{BF}$ interactions finite, then (3) corresponds to the lowest member of the split ground manifold, and hence our $\rho_{B}(x)$ and $\rho_{F}(x)$ are the TG limit of the true ground state partial densities for large but finite repulsions. Nevertheless, in the TG limit the density profiles are labile, since linear combinations of the degenerate ground basis [10] have different density profiles.

Density-density correlations and collective excitation spectrum: The $\mathrm{BB}, \mathrm{FF}$, and $\mathrm{BF}$ density-density correlation functions for this model depend only on $\Psi_{0 M}$, and hence are all the same as that of an ideal Fermi gas or TG gas of $N$ particles apart from normalization; see Eqs. (10) and (11) of [9]. This implies, e.g, that no composite fermions are found (as is the case for a BF mixture on a lattice for particular values of $g_{B F}$ and $g_{B B}$ [11]). The spectrum of collective excitations is given by the poles of the dynamic structure factor, and hence coincides with that of an ideal Fermi gas. For the case of harmonic trapping the spectrum is given by integer multiples of the trapping frequency $\omega$ for both in-phase and out-ofphase modes, in disagreement with [7].

Momentum distributions: Momentum distributions, Fourier transforms of the reduced one-body density matrices with respect to $x-x^{\prime}$, differ greatly between bosons and fermions. Consider first the bosonic one-particle density matrix $\rho_{1 B}\left(x, x^{\prime}\right)$ and momentum distribution $n_{B}(k)$. Using (3) one finds that $\rho_{1 B}$ is proportional to the one-body density matrix of a pure TG gas of $N$ bosons, $\rho_{1 B}\left(x, x^{\prime}\right)=\left(N_{B} / N\right) \rho_{1 T G}\left(x, x^{\prime}\right)$, and hence $n_{B}(k)=\left(N_{B} / N\right) n_{T G}(k)$. Although $\rho_{1 T G}$ is not known analytically, the asymptotics of $n_{T G}(k)$ are known for a homogeneous system of density $\rho_{T G}$, and are given by $n_{T G}(k) \propto|k|^{-1 / 2}$ for $k \ll \rho_{T G}$ [12] and by $n_{T G}(k) \propto|k|^{-4}$ for $k \gg \rho_{T G}$ 13. Determination of $\rho_{1 F}\left(x, x^{\prime}\right)$ and $n_{F}(k)$ is much more difficult. We detail here the derivation for the homogeneous system. Comparing Eq. (3) with $\Psi_{0}$ of the pure TG gas [6] we obtain $\rho_{1 F}\left(x, x^{\prime}\right)=$ $\mathfrak{N}_{F} \int d x_{1} \ldots d x_{N-1}\left|\Psi_{0 M, N-1}\right|^{2} \prod_{1 \leq j \leq N_{B}} \operatorname{sgn}\left(x_{j}\right.$ $x) \operatorname{sgn}\left(x_{j}-x^{\prime}\right) \prod_{1 \leq j \leq N-1} 4 \sin \left(\pi\left(x_{j}-x\right) / L\right) \sin \left(\pi\left(x_{j}-\right.\right.$ $\left.x^{\prime}\right) / L$ ) where $\Psi_{0 M, N-1}$ is $\Psi_{0 M}$ for $N-1$ particles. The determinantal expression $\Psi_{0 M, N-1}=$ $\operatorname{det}\left[e^{i k_{j} x_{\ell}}\right]_{j, \ell=1, \ldots N-1}$, where $k_{j}=-(N-2) \pi / L,-(N-$ 4) $\pi / L, \ldots,(N-4) \pi / L,(N-2) \pi / L$ and some algebra reduce $\rho_{1 F}$ to the sum of a product of onedimensional integrals, of which the first $N_{B}$ involve $b\left(t, x, x^{\prime}\right)=|\sin (\pi(t-x) / L)|\left|\sin \left(\pi\left(t-x^{\prime}\right) / L\right)\right|$ as in a TG gas, and the remaining $N_{F}$ contain 
$f\left(t, x, x^{\prime}\right)=\sin (\pi(t-x) / L) \sin \left(\pi\left(t-x^{\prime}\right) / L\right)$ as in an ideal Fermi gas. Combining the dissimilar factors by the "phase trick" of [7, 14], one finds the final result

$$
\rho_{1 F}\left(x, x^{\prime}\right)=\alpha \int \frac{d \phi}{2 \pi} e^{-i N_{B} \phi} \operatorname{det}_{j, k=1, \ldots N-1}\left[u_{j, k}\left(x, x^{\prime}, \phi\right)\right]
$$

where $\alpha=\left(N_{F} / N L\right) \mathcal{C}\left(\mathcal{N}_{\mathcal{B}}, \mathcal{N}_{\mathcal{F}}\right)$, and $u_{j, k}\left(x, x^{\prime}, \phi\right)=$ $\frac{4}{L} \int_{0}^{L} d t\left[e^{i \phi} \operatorname{sgn}(t-x) \operatorname{sgn}\left(t-x^{\prime}\right)+1\right] \sin (\pi(t-$ $x) / L) \sin \left(\pi\left(t-x^{\prime}\right) / L\right) e^{i 2 \pi t(j-k) / L}$. This can be extended to a harmonic trap.

Ground and excited states for large but finite $B B$ and $B F$ repulsion: Suppose now that the interactions are finite LL interactions [8] $g_{B B} \delta\left(x_{j B}-x_{\ell B}\right)$ and $g_{B F} \delta\left(x_{j B}-\right.$ $\left.x_{\ell F}\right)$. Define dimensionless coupling constants $\lambda_{B B}=$ $\frac{g_{B B}}{2 \hbar} \sqrt{\frac{m}{2 \hbar \omega}}$ and $\lambda_{B F}=\frac{g_{B F}}{2 \hbar} \sqrt{\frac{m}{2 \hbar \omega}}$. The TG limit, to which our ground state (3) corresponds, is $\lambda_{B B} \rightarrow+\infty$ and $\lambda_{B F} \rightarrow+\infty$. As previously noted this state is only one member of a degenerate ground manifold [10], and we now examine how this degeneracy is split when $\lambda_{B B}$ and $\lambda_{B F}$ are large but finite. The exact solutions of the two-body problem are known [15] and the energy eigenfunctions $\Psi_{\nu}\left(x_{B F}\right)$ of the relative motion in a harmonic well are expressible in terms of parabolic cylinder functions $D_{\nu}(\xi)$ [16] where $\xi=x_{B F} \sqrt{\frac{m \omega}{\hbar}}$. The corresponding relative energy eigenvalues are $E_{\nu}=\left(\nu+\frac{1}{2}\right) \hbar \omega$ where $\nu$ is a solution of the transcendental equation $\Gamma\left(\frac{1}{2}(1-\nu)\right) / \Gamma\left(-\frac{1}{2} \nu\right)=-\lambda_{B F}$, and reduce to the harmonic oscillator (HO) quantum numbers $n=0,1,2, \cdots$ in the noninteracting limit. Here we want the opposite limit $\lambda_{B F} \gg 1$. In the TG limit all the eigenfunctions are $\Psi_{n}\left(x_{B F}\right)=A\left(x_{B F}\right) \Psi_{n M}\left(x_{B F}\right)$ where $A$ is a mapping function [10] and the model functions $\Psi_{n M}$ are the odd HO functions $u_{n}$ with $n=1,3,5, \cdots$ so as to vanish at contact. There are two possible mapping functions: $A_{1}=\operatorname{sgn}\left(x_{B F}\right)$ and $A_{2}=+1$, and the ground state is twofold degenerate, with even and odd eigenfunctions $\Psi_{1}^{+}=\operatorname{sgn}\left(x_{B F}\right) u_{1}\left(x_{B F}\right)$ and $\Psi_{1}^{-}=A_{2} u_{1}=u_{1}\left(x_{B F}\right)$ and energy $E_{1}=\frac{3}{2} \hbar \omega$. For large but finite $\lambda_{B F}$ the lowest even eigenfunction is $\Psi_{1}^{+}=D_{\nu_{1}}(|\xi|)$ where $\nu_{1}$ is the solution of the above transcendental equation closest to 1 . $\Psi_{1}^{+}$does not vanish at $x_{B F}=0$ and has an LL cusp [8] there due physically to the delta function interaction and mathematically to the absolute value in its argument [17]. Using gamma function identities and Taylor expansions one finds $\nu_{1}=1-\frac{\lambda_{B F}^{-1}}{\sqrt{\pi}}+O\left(\lambda_{B F}^{-2}\right)$. On the other hand, the lowest odd eigenfunction $\Psi_{1}^{-}$is the lowest odd unperturbed HO eigenfunction $u_{1}$, since $u_{1} \delta\left(x_{B F}\right)=0$. Hence the symmetric solution $\Psi_{1}^{+}$is the ground state and $\Psi_{1}^{-}=u_{1}$ is the first excited state, with excitation energy $\Delta E=\frac{\hbar \omega}{\lambda_{B F} \sqrt{\pi}}+O\left(\lambda_{B F}^{-2}\right)$. More generally, all of the eigenfunctions in the order of increasing energy are $D_{\nu_{1}}(|\xi|), u_{1}, D_{\nu_{2}}(|\xi|), u_{2}, \cdots$, alternately even and odd with $0,1,2, \cdots$ nodes. This is in agreement with SturmLiouville theory and the theorem that the ground state of a Boltzmann or Bose system is real, nonnegative, and hence nodeless.

For arbitrary $N_{B}$ and $N_{F}$ and finite coupling constants the exact solutions are known in the untrapped case only for the special case $\lambda_{B B}=\lambda_{B F}$. However, the above theorem on ground state symmetry has a generalization according to which the ground state is nodeless except for Fermi antisymmetry nodes. It follows that the ground state vanishes only at FF contact points $x_{j B}=x_{\ell F}$, and at all $\mathrm{BB}$ and $\mathrm{BF}$ collision points it has interaction cusps of LL form [8]. By comparison with Eq. (3), one concludes that (3) is the TG limit of the finite-interaction ground state; all other choices [10] of the TG mapping $A$ correspond to excited states. The manner in which the degeneracy of the TG ground manifold splits when $\lambda_{B B}$ and $\lambda_{B F}$ are made large but finite is related to symmetries of all the different mappings [10] yielding the different ground states degenerate in the TG-limit. Consider first a mapping $A^{(0)}$ differing from (2) by omission of all of the factors $\operatorname{sgn}\left(x_{j B}-x_{\ell F}\right)$. The corresponding mapped state is $\Psi_{0}^{(0)}=A^{(0)} \Psi_{0 M}$ where $\Psi_{0 M}$ is the previouslydefined fermionic model ground state. $A^{(0)}$ restores the correct symmetry under BB permutations and introduces the necessary interaction cusps at BB collision points [8], while leaving $\Psi_{0}^{(0)}$ antisymmetric not only under FF permutations, but also locally antisymmetric under BF exchanges, in the sense that $\Psi_{0}^{(0)}$ changes sign as $x_{j B}-x_{\ell F}$ passes through zero [18]. It follows that $\Psi_{0}^{(0)}$ is an eigenstate not only of the TG Hamiltonian $\lambda_{B B} \rightarrow \infty$ and $\lambda_{B F} \rightarrow \infty$, but also of a Hamiltonian with TG BB interactions (i.e., $\lambda_{B B} \rightarrow \infty$ ) but finite $\mathrm{BF}$ interactions, because the nodes at BF collision points "kill" the delta function interactions, and its energy is the same as that in the TG limit. It is not the ground state because of the previously-stated theorem, and in fact it is the top of a ladder of closely-spaced levels extending upward from the ground state, with splittings of order $\frac{\hbar \omega}{\gamma_{B F}}$ between adjacent levels, obtained by restoring more and more collision sgn factors in the mapping function and hence collision cusps in the wave function, thus generating additional downward shifts of order $\frac{\hbar \omega}{\gamma_{B F}}$.

Other soluble models : In addition to the previously discussed Bose-Fermi mixture (which we denote as Model I) we can solve several other models with a similar approach. Model II is a mixture of two noninteracting Fermi gases with interspecies point hard-core repulsions, and model III is a mixture of two Bose gases A and B with inter- and intraspecies hard-core point interactions. Model II could be realized, e.g., by choosing two hyperfine levels of ${ }^{40} \mathrm{~K}$ or ${ }^{6} \mathrm{Li}$ and by tuning to very large and repulsive the interspecies interactions by a confinementinduced Feshbach resonance and analogously model III could be realized by choosing two hyperfine levels of a bosonic alkali atom e.g., $\mathrm{Na}$ or Rb. Models I-III all start from the same model state $\Psi_{M}$, so we only state final 
results, again for harmonic trapping. For both models II and III the density profiles are proportional to those of a TG gas of $N=N_{A}+N_{B}$ bosons, and in model III the momentum distributions for both components are also proportional to those of a $N$-particle TG gas, while for model II the $A$-component momentum distribution $n_{A}(k)$ is equal to $n_{F}(k)$ for model I with $N_{A}$ fermions and $N_{B}$ bosons, and similarly for $n_{B}(k)$ with $A$ and $B$ interchanged. These models have degenerate ground states by the same mechanism as model I.

Finally, we can consider binary mixtures with large, attractive, odd-wave interactions between the two species due to a p-wave Feshbach resonance, in the so-called fermionic Tonks-Girardeau (FTG) limit 2, 19, 20] wherein the fermionic wave function is nonzero with sign change at contact. Model IV is a mixture of two Bose gases $\mathrm{A}$ and $\mathrm{B}$ with no $\mathrm{AA}$ or $\mathrm{BB}$ interactions but an $\mathrm{AB}$ interaction of FTG form, model $\mathrm{V}$ is a mixture of ideal Bose gas B and FTG gas F with FTG BF interactions, and model VI is a mixture of two FTG gases A and B with FTG AB interactions. These models all have the same model state $\Psi_{M}$, a mixture of two dissimilar ideal Bose gases, while the mapping function $A$ has sgn factors for all collisions where an FTG interaction is desired [2, 19, 20]. The momentum distributions for the spatially uniform case on a ring have Lorentzian form as for the FTG gas [21], with the range of the density matrix of each component determined by the sum of the densities of all components (either the same or different) with which it interacts.

We have identified further models with attractive interspecies FTG interactions solvable by mapping from ideal gas mixtures to mixtures of an ideal Fermi gas or TG Bose gas and an ideal Bose gas or FTG Fermi gas, or a mixture of two dissimilar ideal Fermi gases or TG Bose gases, and we believe that this covers all two-component mixture models solvable by mapping functions everywhere \pm 1 , with jumps at contact points which convert Fermi antisymmetry nodes to Bose hard core cusps and Bose-Bose or dissimilar species contact points to infinite FTG attraction discontinuities.

Experimental realizability and applications: Since the TG regime in pure ultracold gases has been reached [4, 5] and p-wave resonances required for the FTG regime have been observed [22], several of these strongly interacting mixture models should be experimentally realizable. The low-lying level structure we have found for Bose-Fermi mixtures might be exploited for quantum computation.

This work was initiated at the Institut Henri PoincaréCentre Emile Borel (IHP) in Paris, during the 2007 workshop "Quantum Gases". We are grateful to the organizers, to the IHP for hospitality and support, and to Jason Ho and Gora Shlyapnikov for helpful suggestions. We are also very grateful to Hrvoje Buljan for pointing out to us the degeneracy of the TG Bose-Fermi ground state. Research of M.D.G. is partially supported by the U.S.
Office of Naval Research, and that of A.M. by the Centre National de la Recherche Scientifique (CNRS).

* Electronic address: girardeau@optics.arizona.edu

$\dagger$ Electronic address: anna.minguzzi@grenoble.cnrs.fr

[1] M. Olshanii, Phys. Rev. Lett. 81, 938 (1998).

[2] B.E. Granger and D. Blume, Phys. Rev. Lett. 92, 133202 (2004).

[3] J.L. Roberts et al., Phys. Rev. Lett. 86, 4211 (2001).

[4] B. Paredes, et al., Nature 429, 277 (2004); T. Kinoshita, T.R. Wenger, and D.S. Weiss, Science 305, 1125 (2004).

[5] T. Kinoshita, T.R. Wenger, and D.S. Weiss, Phys. Rev. Lett. 95, 190406 (2005) and Nature 440, 900 (2006).

[6] M. Girardeau, J. Math. Phys. 1, 516 (1960); M.D. Girardeau, Phys. Rev. 139, B500 (1965), Secs. 2, 3, 6.

[7] A. Imambekov and E. Demler, Ann. Phys. 321, 2390 (2006).

[8] E.H. Lieb and W. Liniger, Phys. Rev. 130, 1605 (1963).

[9] M.D. Girardeau, E.M. Wright, and J.M. Triscari, Phys. Rev. A 63, 033601 (2001).

[10] H. Buljan pointed out to us that the ground state is $2^{\nu}$ fold degenerate where $\nu=\frac{N !}{N_{B} ! N_{F} !}-1$. A real basis for the ground manifold is generated by assigning \pm 1 values of $A\left(X_{B}, X_{F}\right)$ at will to the $N$ ! permutation sectors with the constraint that each mapping $A$ is antisymmetric under permutations of bosons and symmetric under permutations of fermions, guaranteeing that $\Psi=A \Psi_{M}$ will be Bose-symmetric and Fermi-antisymmetric.

[11] M. Lewenstein, L. Santos, M. Baranov, and H. Fehrmann PRL 92, 050401 (2004)

[12] A. Lenard, J. Math. Phys. 5, 930 (1964), H.G. Vaidya and C.A. Tracy, Phys. Rev. Lett. 42, 3 (1979).

[13] A. Minguzzi, P. Vignolo, and M.P. Tosi Phys. Lett. A 294, 222 (2002), M. Olshanii and V. Dunjko,Phys. Rev. Lett. 91, 090401 (2003).

[14] We use that $\sum_{P, Q} \varepsilon(P) \varepsilon(Q) \prod_{j=1}^{N_{B}} B_{j}(P, Q) \prod_{j=N_{B}+1}^{N-1} F_{j}(P, Q)=$ $\mathcal{C}\left(\mathcal{N}_{\mathcal{B}}, \mathcal{N}_{\mathcal{F}}\right) \int \frac{d \phi}{2 \pi} e^{-i N_{B} \phi} \sum_{P, Q} \varepsilon(P) \varepsilon(Q) \prod_{j=1}^{N-1}\left(e^{i \phi} B_{j}(P, Q)+\right.$ $\left.F_{j}(P, Q)\right)$ where $B_{j}=\int d t b\left(t, x, x^{\prime}\right) \exp \left[i\left(P\left(k_{j}\right)-\right.\right.$ $\left.\left.Q\left(k_{j}\right)\right) t\right], F_{j}=\int d t f\left(t, x, x^{\prime}\right) \exp \left[i\left(P\left(k_{j}\right)-Q\left(k_{j}\right)\right) t\right]$ and $\mathcal{C}\left(\mathcal{N}_{\mathcal{B}}, \mathcal{N}_{\mathcal{F}}\right)=N_{B} !\left(N_{F}-1\right) ! /\left(N_{B}+N_{F}-1\right) !$

[15] M.A. Cirone, K. Goral, K. Rzazewski, and M. Wilkens, J. Phys. B 34, 4571 (2001); S. Franke-Arnold, S.M. Barnett, G. Huyet, and C. Saillot, Eur. Phys. J. D 22, 373 (2003).

[16] I.S. Gradshteyn and I.M. Ryzhik, Tables of Integrals, Series, and Products (Academic Press, New York, 1980), pp. 1064-1067.

[17] The $D_{\nu}(y)$ are neither even nor odd, being sums of even and odd hypergeometric functions [16]. They vanish like $y^{\nu} e^{-y^{2} / 4}$ as $y \rightarrow+\infty$ and diverge like $|y|^{-\nu-1} e^{y^{2} / 4}$ as $y \rightarrow-\infty$, but our even solution depends only on $\xi=|y|$.

[18] This antisymmetry is not global, i.e., it is true only if the separation between $x_{j B}$ and $x_{\ell F}$ is infinitesimal.

[19] M.D. Girardeau and M. Olshanii, cond-mat/0309396.

[20] M.D. Girardeau, Hieu Nguyen, and M. Olshanii, Optics Communications 243, 3 (2004).

[21] S.A. Bender, K.D. Erker, and B.E. Granger, Phys. Rev. Lett. 95, 230404 (2005).

[22] C.A. Regal, C. Ticknor, J.L. Bohn, and D.S. Jin, Phys. Rev. Lett. 90, 053201 (2003). 